\title{
A note on the effect of the fiber curvature on the micromechanical behavior of natural fiber reinforced thermoplastic composites
}

\author{
M. A. Escalante-Solís, A. Valadez-González, P. J. Herrera-Franco* \\ Unidad de materiales Centro de Investigación Científica de Yucatán Calle 43 \# 130, Col. Chuburná de Hidalgo, C.P. \\ 97200 Mérida, Yucatán, México
}

Received 29 May 2015; accepted in revised form 5 August 2015

\begin{abstract}
To better understand the role of the fiber curvature on the tensile properties of short-natural-fiber reinforced composites, a photoelastic model and a finite element analysis were performed in a well characterized henequen fiber-high density polyethylene composite material. It was hypothesized that the angle of orientation of the inclusion and the principal material orientation with respect to the applied load was very important in the reinforcement mechanics. From the photoelastic and finite element analysis it was found that the stress distribution around the fiber inclusion was different on the concave side from that observed on the convex side and an efficient length of stress transfer was estimated to be approximately equal to one third the average fiber length. This approach was used to predict the short-natural-fiber reinforced composite mechanical properties using probabilistic functions modifications of the rule of mixtures models developed by FukudaChow and the Fukuda-Kawata. Recognizing the inherent flexibility that curves the natural fibers during processing, the consideration of a length of one third of the average length $\bar{l}$ should improve the accuracy of the calculations of the mechanical properties using theoretical models.
\end{abstract}

Keywords: reinforcement, natural fibers, fiber critical length, micromechanics, interphase shear strength

\section{Introduction}

Cellulosic fibers like flax, hemp, kenaf, henequen, sisal, jute, coconut, coir, kapok, banana and many others have been used as reinforcement agents of different thermosetting and thermoplastic resins. Unlike the traditional engineering fibers, e.g. glass and carbon fibers, these lignocellulosic fibers are able to impart the composite high specific stiffness and strength, they have a desirable fiber aspect ratio and a high degree of flexibility, recyclability and biodegradability, they are non-abrasive to the processing equipment, non-irritating to the skin, no other health hazards, they are readily available from natural sources, and more importantly, they have a low cost per unit volume basis [1,2].
The problem of fiber-polymeric matrix adhesion has been approached modifying either the fiber surface, or the matrix to ensure the stress transfer, necessary for the effectiveness of the composite material. It follows that the efficiency of load transfer from matrix to fiber in a composite is not only strongly related to the optimum mechanical properties of the constituent components but also to the interfacial parameters, including factors such as: the interfacial shear strength, the interfacial toughness, the matrix shrinkage pressure on the fiber, and the interfacial coefficient of friction [3, 4].

Effective analytical micromechanical models have been developed for synthetic short fiber-reinforced polymers [5]. The most utilized and known theories

\footnotetext{
${ }^{\text {*Corresponding author, e-mail: pherrera@cicy.mx }}$

(C) BME-PT
} 
for tensile properties are the rule of mixtures (ROM), the Cox model, the Halpin-Tsai model, and the Bowyer-Bader model [6-8]. The ROMs are very simple models using fiber and matrix sum of volume weighted properties to predict composite properties. ROM can be parallel or series. The Hirsch model is a combination of parallel and series ROM. The HalpinTsai model considers matrix-to-fiber properties ratio instead of sum of volume-weighted properties. The Cox model suggests that stress in the fiber is proportional to the difference between fiber strain and the strain that the matrix would have if there were no fibers. The Bowyer-Bader model considers the contribution of fibers below and above critical fiber length. More recently, Nairn proposed a generalized shear-lag analysis model to calculate the tensile elastic modulus of the composite. Nairn extended the optimal shear-lag analysis to a generalized case by enabling the shear stresses to be described by shape functions. He also extended the capabilities of the generalized shear-lag analysis to include an imperfect interface by the addition of an interface parameter $D_{\mathrm{s}}[9]$. Nairn's model considers the interfacial quality explicitly; however, most models use constants to fit the experimental measurements. Some authors that have used analytical modeling for natural fiber-reinforced (or filled) polymers, incorporated a reinforcement efficiency factor. For example, Simonsen used factors that varied between 0.61 and 0.85 , depending on the polymer matrix to model the bending modulus of elasticity of wood fiber-filled thermoplastics made with three different matrices: polypropylene, polyethylene, and polystyrene. A loss factor for fiber orientation has also been used to fit the model to experimental values. Orientation factors for tensile strength found varied from 0.1 to 0.36 . The loss factor for stress transfer efficiency was used for the composite modulus of elasticity varied from 0.42 to 0.51 for injection molded jute/polypropylene composites as a function of fiber volume fraction using ROM [10, 11]. Fiber/matrix interfacial shear stress was obtained from single fiber pull-out tests and was incorporated into the tensile strength model. A few other authors have incorporated values for the critical length $\left(L_{\mathrm{c}}\right)$ and fiber matrix-adhesion strength $(\tau)$ for hemp single fiber/ polypropylene composites [12] and Herrera-Franco and Valadez-González [13] used the single fiber fragmentation tests together with pull-out tests on henequen/polyethylene single fiber specimens and found that the critical length and the fiber aspect ratio, varied with fiber/matrix adhesion quality and interfacial shear strength.

Fukuda and Kawata [14] developed a theory for the Young's modulus of short-fiber reinforced composites with variable fiber length and orientation. Later on, Fukuda and Chou [15] adopted the basic probabilistic approach of Fukuda and Kawata [14] to develop a theory for the modulus of short-fiber reinforced composites with variable fiber length and orientation. Summarizing it can be said that the role of the fiber reinforcement is considered in these model using an efficiency factor (or loss factor), together with an orientation factor. The first factor is related to the fiber length, either above or below the fiber critical length $L_{\mathrm{c}}$, and sometimes estimated from the interfacial shear strength. The second factor is equal to one for unidirectionally oriented fibers and less than one for randomly oriented fibers depending on the angle distribution of the fibers.

Little attention has been paid to the fact that natural fibers are flexible and that depending on their initial length before processing and the processing method used, their final shape will be distorted with shapes other that the stiff, straight fibers upon which all the micromechanical models were developed.

The aim of this paper was the study of the role of the fiber curvature on the prediction of the tensile properties of short-natural-fiber reinforced composites for a well-defined fiber-matrix adhesion system. Emphasis on the flexible behavior of the natural fibers, therefore, fiber length and orientation and its effect on its stress transfer capability and the mechanical properties of the constituents will be discussed. The discussion is built around a well characterized and controlled fiber-matrix system, namely, a high density polyethylene (HDPE) matrix reinforced with henequen fibers (Agave fourcroydes).

Although the fibers, as a result of the processing method of the composite, may exhibit a curvature with shapes such as $C, D, S$, etc., the U-shape was proposed in this paper for the simplicity of the analysis and as the basis to analyze the effect of the other shapes mentioned before. In any case, the approach to handle the fiber curvature will be discussed in the section 7.1. 


\section{Fiber-matrix adhesion and experimental procedures}

The optimization of interfacial bond between a fiber and a polymer matrix is an important aspect with respect to the optimal mechanical performance of fiber reinforced composites in general, and durability in particular. Since the fibers and matrices are chemically different, strong adhesion at their interfaces is needed for an effective transfer of stress and bond distribution throughout an interface. A good compatibility between cellulose fibers and nonpolar matrices is achieved from polymeric chains that will favor entanglements and inter-diffusion with the matrix $[13,16]$. Several approaches have been explored, such as physical treatments by corona or plasma, and chemical grafting with molecules and macromolecules which display a good compatibility with the matrix and introduce surface hydrophobicity. The chemical modification by coupling agents susceptible to react with the fibers and the matrix constitutes a particularly astute way of controlling the quantity and the nature of the groups present at their surface. Moreover, it creates covalent bridges between the fibers and the matrix which ensure the best mechanical properties for the composite [17]. The physical and mechanical properties of the henequen fiber and the HDPE are listed in Table 1 [13]. The fiber surface properties were modified to enhance the physicochemical interactions at the fibermatrix interphase. They are first treated with a $\mathrm{NaOH}$ aqueous solution alkaline treatment which has two effects on the fiber: (1) it increases the surface roughness that results in a better mechanical interlocking; and (2) it increments the amount of cellulose exposed on the fiber surface, thus increasing the number of possible reaction sites.

In this paper, the following materials were used: as a matrix, high density polyethylene (HDPE) and as reinforcement, henequen fibers (Agave fourcroydes) with an average diameter of $180 \mu \mathrm{m}$ approximately.

In order to enhance the fiber-matrix adhesion two surface pre-treatments were used: first, a silane coupling agent was used and second, a matrix pre-

Table 1. Physical and mechanical properties of the constituents of composite

\begin{tabular}{|l|c|c|c|c|}
\hline Material & $\begin{array}{c}\boldsymbol{\sigma} \\
{[\mathbf{M P a}]}\end{array}$ & $\begin{array}{c}\mathbf{E} \\
{[\mathbf{M P a}]}\end{array}$ & $\begin{array}{c}\text { Poisson } \\
\text { ratio, } \boldsymbol{v}\end{array}$ & $\begin{array}{c}\text { Equivalent diameter } \\
{[\mathbf{m m}]}\end{array}$ \\
\hline Henequen & 604 & 13200 & 0.33 & 0.18 \\
\hline HDPE & 28 & 792 & 0.30 & - \\
\hline
\end{tabular}

impregnation on the fiber. The henequen fibers were treated with a $2 \% \mathrm{NaOH}$ aqueous solution for 1 hour at $25^{\circ} \mathrm{C}$, washed with distilled water and dried at $60^{\circ} \mathrm{C}$ for $24 \mathrm{~h}$, after they were surface modified.

The henequen fibers were also treated with vinyltris(2-ethoxymethoxy) silane coupling agent and dicumyl peroxide. The peroxide and silane coupling agent were deposited on the surface of the fiber from a methanol/water solution (90:10) adjusted to a pH 3.5 with acetic acid at $25^{\circ} \mathrm{C}$. The weight concentration was $1 \%$ silane and $0.5 \%$ peroxide with respect to the fiber. Then the fibers were dried and cured at $60^{\circ} \mathrm{C}$ during $24 \mathrm{~h}$.

The fiber-surface silanization opens the way to the rational use of silane coupling agents to functionalize the surface of organic materials. In some cases, optimal conditions can be established thus favoring the substrate condensation reaction and limiting the self-condensation of the silanol groups that produce polysiloxanes. This is particularly interesting, since the grafting efficiency (both in terms of product configuration at the surface and quantity of adsorbed molecules) can be modulated, depending on the envisaged extent of the fiber-surface modification $[18,19]$.

After the surface modifications, the fibers were preimpregnated with a solution of HDPE-xylene to ensure a better wetting of the fibers with the polymer. Fiber preimpregnation allows a better fiber wetting which in a normal fiber-polymer mixing procedure would not be possible because of the high polymer viscosity, thus, it enhances the mechanical interlocking between fiber and matrix. The nomenclature used for the different fiber surface treatments and the expected adhesion mechanisms are described in Table 2.

The composite laminates were prepared using a fiber volume fraction of $20 \% \mathrm{w} / \mathrm{w}\left(V_{\mathrm{f}}=0.1237\right)$ calculated

Table 2. Nomenclature used for different fiber surface treatments

\begin{tabular}{|l|l|l|}
\hline \multicolumn{1}{|c|}{$\begin{array}{c}\text { Type of } \\
\text { bonding }\end{array}$} & Keyword & \multicolumn{1}{c|}{ Description of treatment } \\
\hline \multirow{2}{*}{$\begin{array}{l}\text { Mechanical } \\
\text { bonding }\end{array}$} & FIB & Fiber without any treatment \\
\cline { 2 - 3 } & FIBNAPRE & $\begin{array}{l}\text { Fiber treated with a NaOH aque- } \\
\text { ous solution }\end{array}$ \\
\cline { 2 - 3 } & $\begin{array}{l}\text { Fiber treated with a NaOH aque- } \\
\text { ous solution and then pre-impreg- } \\
\text { nated with dissolved HDPE }\end{array}$ \\
\hline $\begin{array}{l}\text { Mechanical plus } \\
\text { chemical bond- } \\
\text { ing }\end{array}$ & FIBNASIL & $\begin{array}{l}\text { Fiber treated with a NaOH aque- } \\
\text { ous solution and then with a } \\
\text { silane coupling agent }\end{array}$ \\
\hline
\end{tabular}


considering the fiber density equal to $1.7 \mathrm{~g} / \mathrm{cm}^{3}$. The value of the shear modulus of the matrix, $G_{\mathrm{m}}$, was calculated using the expression $G_{\mathrm{m}}=E_{\mathrm{m}} /\left(2\left(1+v_{\mathrm{m}}\right)\right)$ where $E_{\mathrm{m}}$ and $v_{\mathrm{m}}$ are the Young's modulus and Poisson's ratio of the matrix, respectively [14]. The fiber length used for the composite fabrication was equal to $15 \mathrm{~mm}$.

\section{Photoelastic model and resin calibration}

To know the stress distribution around a curved inclusion in an infinite media, when the material was subjected to a tensile load, a photoelastic analysis was performed. It is convenient to mention that this photoelastic as well as a finite element analysis, to be mentioned latter, were performed not to measure the actual stress at the fiber-matrix interface, but rather to identify the distribution of the stress components around the curved fiber (inclusion). The resin selected to make the model was an epoxy, Bisphenol A, DER 331 from DOW Chemical because of its excellent photoelastic properties. The curing agent used was an aliphatic amine, Ancamine 1784 from Air Products and Chemicals, Inc., using 60\% w/w ratio with the resin. After mixing and degassing, the resin was poured in silicon mold to fabricate a disk with a diameter $D$ equal to $44.6 \mathrm{~mm}$. To simulate a curved fiber inclusion, a piece of copper wire of $0.32 \mathrm{~mm}$ of diameter was used. The composite single inclusion sample was an ASTM standard tensile test dog bone shaped specimen. It was also casted in a silicon mold. First, the resin was poured to fill half the mold cavity and when the resin started to gel, the wire was carefully placed in the cavity and then the mold was filled with the resin and allowed to cure at room temperature for 48 hours.

The principal-stress difference $\left(\sigma_{1}-\sigma_{2}\right)$ at each point in the model is proportional to the induced birefringence at the point. The constant of proportionality was determined using a well-known method of a disk loaded in diametric compression. The isochromatic pattern is related to the stress system by the stress-optic law (Equation (1)):

$\sigma_{1}-\sigma_{2}=\frac{N f_{\sigma}}{t}$

where $f_{\sigma}$ is the stress-optical coefficient, a constant that depends upon the model material and the wavelength of light employed, $t$ is the model thickness, and $N$ is the relative retardation of rays forming the pat- tern. The term $N$ is also known as an isochromatic fringe order. The state of stress at the center of the disk is known [20] and substituting in Equation (1), the stress-optical coefficient fo is given by Equation (2):

$f_{\sigma}=\frac{8 P}{\pi D N}$

where $P$ is the diametral compression force and $D$ is the diameter of the disk.

Then, the maximum shear stress in the plane is obtained relating the fringes to fringe order by Equation (3):

$\tau_{\max }=\frac{\sigma_{1}-\sigma_{2}}{2}=\frac{N f_{\sigma}}{2 t}$

\section{Finite element analysis}

As mentioned previously, a finite element analysis was also performed together with the photoelastic analysis. Figure 1 shows the finite element model and the boundary conditions used for the single curved fiber inclusion. A commercial finite element code was used (NISA Ver. 15.0) using rectangular four-node isoparametric elements. A total of 20706 nodes and 20400 elements were used and the material properties were supposed to be homogeneous and isotropic and an elastic plane stress analysis was assumed within the framework of small displacement theory. Perfect adhesion was assumed at the fibermatrix interphase, that is, only two faces are considered, the fiber and the matrix. The material properties used were: for the matrix, $E_{\mathrm{m}}=1032 \mathrm{MPa}$, Poisson's ratio $v_{\mathrm{m}}$ equal to 0.38 and the inclusion, $E_{\mathrm{f} 1}=11.772 \mathrm{GPa}, E_{\mathrm{f} 2}=1.1772 \mathrm{GPa}$ Poisson's ratio $v_{\mathrm{f}}$ equal to 0.35 . The shear modulus in both materials was calculated using $G=E /(2(1+v))$. Special care was taken during meshing to avoid (or, at least, to reduce to a minimum) distorted elements, particularly at the ends of the inclusion.

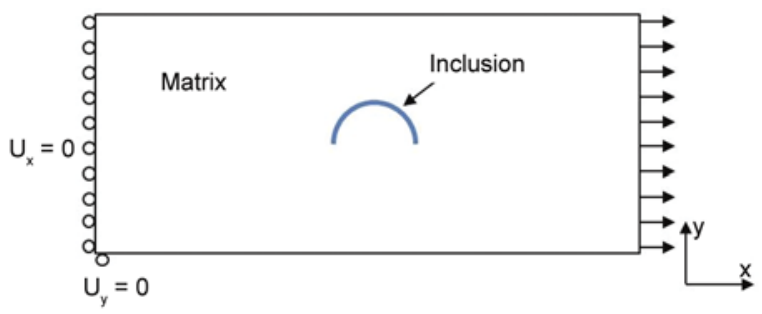

Figure 1. Finite element model and the boundary conditions used to for the single curved fiber inclusion 


\section{Fiber-matrix adhesion characterization and the interphase}

The concept of interphase is the geometrical surface of the classic fiber-matrix contact as well as the region of finite volume extending therefrom, wherein the chemical, physical and mechanical properties vary either continuously or in a stepwise manner between those of the bulk fiber and matrix material. Experimental methods for characterization of the fiber-matrix interface are based on the measurement of the adhesion of a single fiber. The fiber-fragment critical length and the interfacial shear strength can be measured using the Single fiber Fragmentation Test (SFFT). This technique consists of a single fiber embedded in a polymer matrix dog bone shaped specimen tensile specimen. During application of the load, the tensile stress in the fiber increases and fragmentation occurs at points where its tensile strength $\sigma_{\mathrm{f}}$ is reached. This fragmentation process continues as the applied stress $\sigma_{0}$ is increasing. At some point, the fiber fragments are so short that the stress transferred to the fiber through the interface is not enough to cause any further fiber failure. When this happens, it can be said that the critical fiber fragment length $l_{\mathrm{c}}$ has been reached. To a first approximation, the interfacial shear strength $(\tau)$ is calculated from a simple equation obtained from a force balance on a fragment of fiber and, recognizing the random nature of the fiber fragmentation process, the expression is given by Equation (4) [21]:

$$
\tau=\frac{\sigma_{\mathrm{f}}}{2 \eta} \Gamma\left(1-\frac{1}{\alpha}\right)
$$

where $\sigma_{\mathrm{f}}$ is the tensile strength of the fiber, $\alpha$ and $\eta$ are Weibull scale and shape parameters and $\Gamma$ is the Gamma function.

\section{Modelling of micromechanical properties}

The rule of mixtures was also modified by FukudaChou [15] to predict the strength of a fiber reinforced composite when both, the fiber length and orientation varies. For a continuous unidirectional fiber developed using the assumption of equal strain in both matrix and fiber, the rule of mixtures is expressed by Equation (5):

$\sigma_{\mathrm{cu}}=\sigma_{\mathrm{fu}} V_{\mathrm{f}}+\sigma_{\mathrm{m}}\left(1-V_{\mathrm{f}}\right)$ where $\sigma_{\mathrm{cu}}$ and $\sigma_{\mathrm{fu}}$ represent the ultimate strength of the composite and fiver respectively, $V_{\mathrm{f}}$ denotes the fiber volume fraction $\sigma_{\mathrm{m}}$ is the matrix strength upon failure of the composite. In the case of short unidirectional fibers, is expressed by Equation (6):

$$
\sigma_{\mathrm{cu}}=\sigma_{\mathrm{fu}} V_{\mathrm{f}} F\left(l_{\mathrm{d}} / \bar{l}\right)+\sigma_{\mathrm{m}}\left(1-V_{\mathrm{f}}\right)
$$

where the factor $F\left(l_{\mathrm{c}} / \bar{l}\right)$ is added to consider the effect of the fiber length $l_{\mathrm{c}}$ and $\bar{l}$ represent the critical length and the average fiber length respectively. If a constant uniform shear stress and a uniform fiber length are assumed, this factor is given by Equation (7):

$F\left(l_{\mathrm{c}} / \bar{l}\right) \begin{cases}=1-\frac{l_{\mathrm{c}}}{2 \bar{l}} & \left(\bar{l}>l_{\mathrm{c}}\right) \\ =\frac{\bar{l}}{2 l_{\mathrm{c}}} & \left(\bar{l}<l_{\mathrm{c}}\right)\end{cases}$

If the fiber length is not uniform, Equation (6) must be modified. Considering the manufacturing processes used for composite materials, there is a variation, not only of the fiber length but of the fiber orientation too. In the case of randomly oriented fibers, the rule of mixtures is further modified as shown by Equation (8):

$\sigma_{\mathrm{cu}}=\sigma_{\mathrm{fu}} V_{\mathrm{f}} F\left(l_{\mathrm{c}} / \bar{l}\right) C_{0}+\sigma_{\mathrm{m}}\left(1-V_{\mathrm{f}}\right)$

where $C_{0}$ is the factor of fiber orientation. The importance of the factors $F\left(l_{\mathrm{c}} \bar{l}\right)$, and $C_{0}$, are important for the discussion in this paper. Fukuda and Chou [15] also proposed the expansion of the concept of "critical zone' to predict the strength of composites reinforced with randomly fibers reinforced with short fibers with variable fiber orientation and length. The critical zone is defined by means of a pair of planes separated by a distance $\beta \bar{l}$, where $\beta$ is a constant value less than one and $\bar{l}$, is the average length of the fiber crossing a plane normal to the tensile stress applied and aligned with the fiber. The length and orientation of the fiber were considered introducing a probability density function of the fiber length $h(l)$, together with probability density function of fiber orientation $g(\theta)$, and then, the strength of the composite material was also given in the form of a probability density function by Equation (9): 


$$
\begin{array}{r}
\sigma_{\mathrm{cu}}=\sigma_{\mathrm{fu}} V_{\mathrm{f}} \int_{\theta_{0}}^{\pi / 2} g(\theta) \cos \theta \mathrm{d} \theta \int_{0}^{\theta_{0}} g(\theta) \cos ^{3} \theta d \theta \cdot \int_{\beta \overline{\mathrm{I}}}^{\infty}\left[\int_{0}^{\theta_{0}}\left(1-\frac{\beta \bar{l}}{l \cos \theta}\right) g(\theta) \mathrm{d} \theta\right] h(l) \mathrm{d} l . \\
\cdot\left[\int_{\beta 1}^{1_{\mathrm{c}}} \frac{l}{2 l_{\mathrm{c}}} h(l) \mathrm{d} l+\int_{\mathrm{l}_{\mathrm{c}}}^{\infty}\left(1-\frac{l_{\mathrm{c}}}{2 l}\right) h(l) \mathrm{d} l\right]+\sigma_{\mathrm{m}}\left(1-V_{\mathrm{f}}\right)
\end{array}
$$

This is a general expression for the strength of a short fiber reinforced composite. In this case, to analyze a particular composite material it is necessary to know the functions $g(\theta)$ and $h(l)$ as well as $\sigma_{\mathrm{fu}}, \sigma_{\mathrm{m}}, V_{\mathrm{f}}$ and $l_{\mathrm{c}}$. In the case of a unidirectional and uniform fiber length (case 1), this expression was reduced to Equations (10) and (11):

$$
\begin{array}{r}
\sigma_{\mathrm{cu}}=\sigma_{\mathrm{fu}} V_{\mathrm{f}}(1-\beta)\left(1-\frac{l_{\mathrm{c}}}{2 \bar{l}}\right)+\sigma_{\mathrm{m}}\left(1-V_{\mathrm{f}}\right) \\
(\bar{l}>l \mathrm{c}) \\
\sigma_{\mathrm{cu}}=\sigma_{\mathrm{fu}} V_{\mathrm{f}}(1-\beta) \frac{l_{\mathrm{c}}}{2 \bar{l}}+\sigma_{\mathrm{m}}\left(1-V_{\mathrm{f}}\right) \\
(\bar{l}<l \mathrm{c})
\end{array}
$$

In the case of unidirectional distributed fiber length (case 2), $\beta \rightarrow 0$, we get Equation (12):

$$
\begin{array}{r}
\sigma_{\mathrm{cu}}=\sigma_{\mathrm{fu}} V_{\mathrm{f}}\left[\int_{0}^{l_{\mathrm{c}}} \frac{l}{2 l_{\mathrm{c}}} h(l) \mathrm{d} l\right. \\
\left.+\int_{\mathrm{I}_{\mathrm{c}}}^{\infty}\left(1-\frac{l_{\mathrm{c}}}{2 l}\right) h(l) \mathrm{d} l\right]+ \\
+\sigma_{\mathrm{m}}\left(1-V_{\mathrm{f}}\right)
\end{array}
$$

and in the case of random orientation, uniform fiber length, and longer than the critical length we get Equation (13):
If $C_{0}$ is defined, for a constant value of $g(\theta)=2 / \pi$, as shown by Equation (14):

$$
\begin{aligned}
C_{0}= & \frac{4}{\pi^{2}} \frac{2+\beta^{2}}{3}\left(1-\beta^{2}\right)^{1 / 2} . \\
& \cdot \frac{2}{\pi}\left[\cos ^{-1} \beta-\frac{1}{2} \beta \log \frac{1+\left(1-\beta^{2}\right)^{1 / 2}}{1-\left(1-\beta^{2}\right)^{1 / 2}}\right]
\end{aligned}
$$

and in the limiting case, $\beta \rightarrow \infty, C_{0} \rightarrow 0.27$. Figure 2 shows a plot of $C_{0}$ as a function of $\beta$.

In the case of the elastic modulus of the short fiber reinforced composites, Fukuda and Kawata [14] analyzed the load transfer mechanism from fiber to matrix in a single fiber model using the theory of elasticity, assuming that the interactions between fibers were negligible, and expressed as a relative modulus value $K_{\mathrm{c}}$ with respect to the matrix modulus by Equation (15):

$$
K_{\mathrm{c}}=\frac{E_{\mathrm{f}}}{E_{\mathrm{m}}} R_{0} C_{\mathrm{a}} V_{\mathrm{f}}+\left(1-V_{\mathrm{f}}\right)
$$

where $R_{0}$ is given by Equation (16) and $C_{\mathrm{a}}$ by Equation (17):

$\sigma_{\mathrm{cu}}=\sigma_{\mathrm{fu}} V_{\mathrm{f}}\left(1-\frac{l_{\mathrm{c}}}{2 l}\right) \int_{\theta_{0}}^{\pi / 2} g(\theta) \cos \theta \mathrm{d} \theta \int_{0}^{\theta_{0}} g(\theta) \cos ^{3} \theta \mathrm{d} \theta \int_{0}^{\theta_{0}}\left(1-\frac{\beta \bar{l}}{\cos \theta}\right) g(\theta) \mathrm{d} \theta+\sigma_{\mathrm{m}}\left(1-V_{\mathrm{f}}\right)$
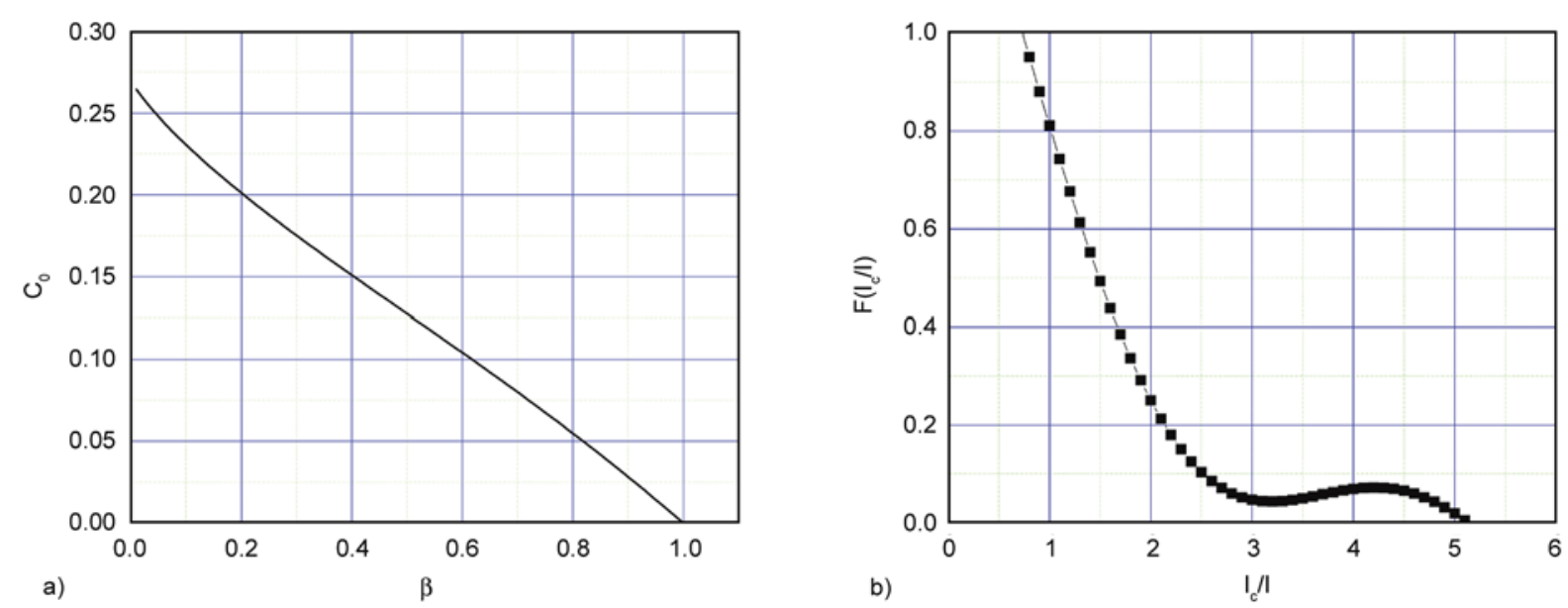

Figure 2. (a) Orientation factor of the fiber $C_{0}$ for random fiber orientation; (b) effect of fiber length on composite strength 
$R_{0}=\frac{-\frac{2}{W_{\mathrm{f}}} \frac{\pi}{3+2 \nu-\nu^{2}} \int_{0}^{\infty}\left(l \int_{0}^{1} \bar{\sigma}_{1}(u) \mathrm{d} u\right) h(l) \mathrm{d} l}{\frac{E_{\mathrm{f}}}{E_{\mathrm{m}}}}$

$C_{\mathrm{a}}=\int_{0}^{\pi / 2} g(\theta) \cos \theta \mathrm{d} \theta \int_{0}^{\theta_{0}} f(\theta) g(\theta) \cos \theta \mathrm{d} \theta$

It should be noted that $K_{\mathrm{c}}$ is independent of the fiber length. When the short fibers are unidirectionally aligned, in the $x$-direction, $g(\theta)$ converts into the Dirac delta value $\delta$ which possess a singularity at $\theta=0$, and $C_{\mathrm{a}}$ is equal to unity. When the fibers are randomly oriented, $C_{\mathrm{a}}$ is less than one and when the fibers are unidirectionally aligned, equal to one. $C_{\mathrm{a}}$ is regarded as the coefficient of reduction of Young's modulus. $R_{0}$ shows the degree of Young's modulus caused by the length of the fiber. When $\alpha_{0}=\pi / 2$, the fiber distribution is random $C_{\mathrm{a}}$ has a value equal to 0.23 .

\section{Results and discussion}

Figure 3 shows photographs of the fibers in the fibers inside the composite materials, for each of the different fiber surface treatments. The curvature of each fiber was represented with the $A / L$ ratio. Here $A$ is the amplitude of the curve and $L$ is the fiber span from end to end. When $A$ tends to zero, the fiber approaches a straight line, that is, the curvature decreases. Typically, fiber fragment lengths are measured using microscopy techniques after dissolving the matrix. However, in this case, these photographs were taken to show that there are fibers whose shape is not straight and any dissolution and handling might change such curved shape. This curvature exhibited by the fibers, is especially noticeable in photographs of FIB, FIBNA and FIBNAPRE composites. The fiber fragments from FIBNASIL do not show a noticeable curvature.

The values of interfacial shear strength, and fiberfragment critical length and tensile properties as a function of the different fiber surface treatments obtained from the SFFT are shown in Table 3. When the fiber surface topography was modified with the alkaline treatment, (FIBNA), a 10\% increase of IFFS was noticed. When the surface-modified fiber was pre-impregnated, a 50\% increase was observed. The treatment with the silane coupling agent results in more impressive results. In the case of FIBNASIL (a combination of mechanical and chemical bonding mechanisms) results in an IFFS increase of more than $160 \%$.

The importance of the chemical bonding is notorious as observed from the higher IFFS increments. Similar observations can be made with the fiber-fragment critical lengths, instead of increments, shorter lengths or fiber aspect ratios are observed for stronger IFFS values. One of the advantages of the single fiber fragmentation test is that the experimental parameter which is actually measured in the test is the fiber-fragment critical length [21, 22].

Optimum mechanical properties in composite materials are strongly related to the efficiency of load transfer. The critical length $l_{\mathrm{c}}$ in composites is a parameter which is an indicator of the amount of stress transferred to the fiber: a fiber whose aspect ratio $s=l / d_{\mathrm{f}}$ (where $d_{\mathrm{f}}$ is the fiber diameter) is much greater than the critical aspect ratio $s_{\mathrm{c}}=l_{\mathrm{c}} / d_{\mathrm{f}}$ strengthens the material, while a fiber whose aspect ratio is much smaller than the critical aspect ratio is more likely to weaken the material $[23,24]$. Therefore, a good understanding of the factors which influence the critical aspect ratio in order to design optimum continuous or short-fiber composites either is impor$\operatorname{tant}$ [25-29].

The effect of the interfacial quality between henequen short, randomly oriented in a matrix of HDPE and its effect on the tensile strength of the composite for various surface treatments is shown in Table 3.

Again, the effect of fiber surface morphology modification results in increments of approximately $11.7 \%$, however, the effect of chemical bonding is

Table 3. Fiber critical length, tensile strength, fiber aspect ratio and interfacial shear strength obtained for the different surface treatments $\left({ }^{*}\right.$ Tensile strength increase [\%] with fiber surface treatment with respect to FIB)

\begin{tabular}{|c|c|c|c|c|c|}
\hline \multirow{2}{*}{$\begin{array}{c}\text { Fiber surface } \\
\text { treatment }\end{array}$} & \multicolumn{3}{|c|}{ Micromechanical results } & \multicolumn{2}{|c|}{ Macromechanical results } \\
\hline & $\begin{array}{c}\text { Critical length } \\
{[\mathrm{mm}]}\end{array}$ & $\begin{array}{c}\text { Fiber aspect ratio } \\
l_{\mathrm{c}} / \mathbf{d}_{\mathrm{f}}\end{array}$ & $\begin{array}{c}\text { IFSS SFFT } \\
\text { [MPa] }\end{array}$ & $\begin{array}{c}\text { Tensile strength } \\
\text { [MPa] }\end{array}$ & $\begin{array}{c}\text { Young's modulus } \\
\text { [MPa] }\end{array}$ \\
\hline FIB & 12.96 & 72.00 & 5.4 & 20.80 & 891.00 \\
\hline FIBNA & 9.25 & 51.38 & $6.0(11 \%)^{*}$ & $21.00(1 \%)^{*}$ & 945.00 \\
\hline FIBNAPRE & 6.00 & 33.33 & $9.2(70 \%)^{*}$ & $23.25(12 \%)^{*}$ & 945.00 \\
\hline FIBNASIL & 3.50 & 19.44 & $16.0(196 \%)^{*}$ & $27.00(30 \%)^{*}$ & 873.00 \\
\hline
\end{tabular}




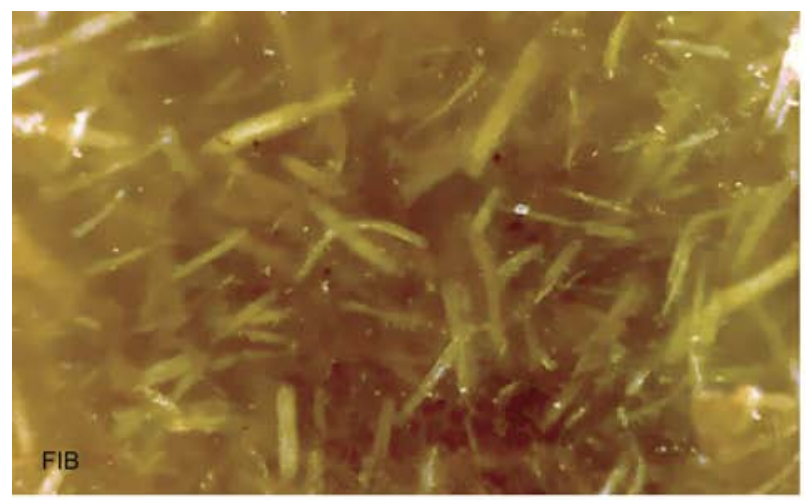

a)

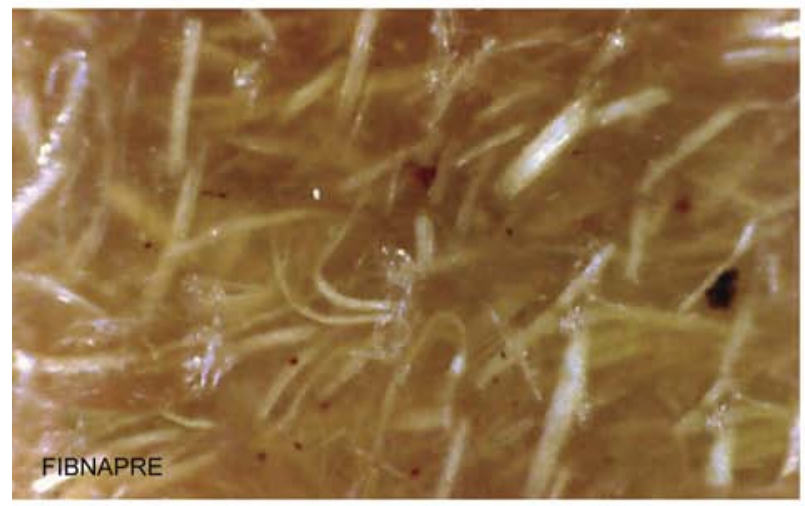

c)

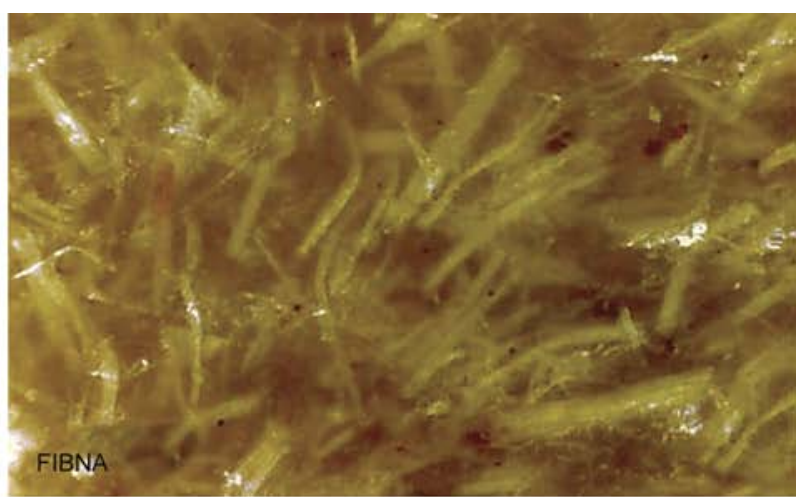

b)

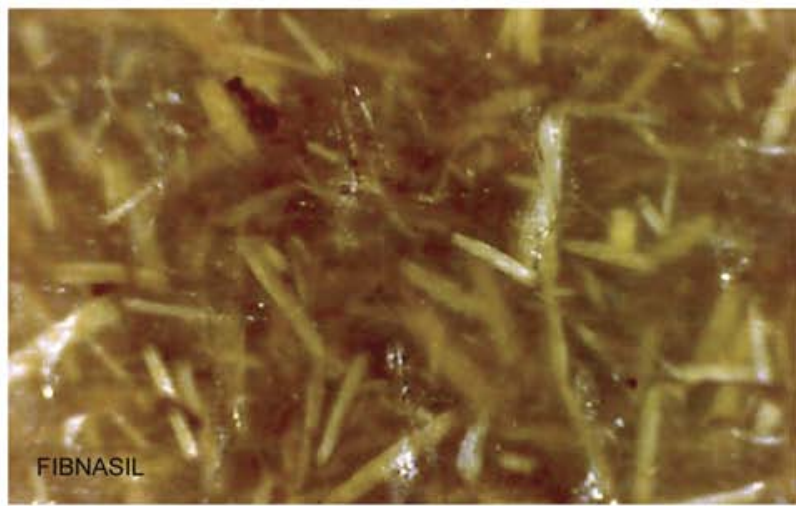

d)

Figure 3. Photographs of fibers inside the composite laminates of FIB, FIBNA, FIBNAPRE and FIBNASIL showing the curved fibers, especially for the first three cases

close to a $30 \%$, that is, three times higher than the mechanical bonding. It should be noticed that the increments of IFSS and tensile strength for each fiber surface treatment with respect to FIB are shown in parenthesis in Table 3. It should also be noticed that the improvements in the IFSS measured obtained with the fiber surface treatments are not reflected in the same increments in the macromechanical properties. The measured values for Young's modulus, on the other hand, were not sensitive to the fiber surface treatments as demonstrated by previous reports [7].

\subsection{Analysis of stress transfer in a curved fiber}

The optimum mechanical properties in composite materials are strongly related to the load transfer efficiency between fiber and matrix. This is especially true in the case of polymeric matrices which are viscoelastic at all temperatures [22]. Other factors that govern the intrinsic properties include fiber architecture, fiber geometry, fiber orientation, packing arrangement and fiber volume fractions, and fibermatrix quality, determine many composite properties, particularly mechanical properties. Additionally, the complex state of stress near fiber fragment ends make the modelling of the mechanical behavior of a natural fiber reinforced composite extremely difficult. One parameter that is usually omitted in most of the micromechanical models is the flexibility of the fiber that results in bent or twisted fibers after processing of the composite. A natural fiber will easily bend in the composite as a result of the high shear stresses resulting from the extrusion and/or injection molding process. With this in mind it is reasonable to think that the micromechanical models require an adjustment factor that takes into account the presence of curvature of the natural fiber to be able to more precisely estimate the mechanical properties of the composite. Although there are reports in the literature that consider other factors that contribute to the theoretical, these results do not conform to the experimental values [25-29]. Furthermore, the structure and composition of a natural fiber allows the formation of failure surfaces because of their microfibrillar and hollow nature and to the irregular shape of their cross section. Also, it should be remembered that henequen fiber itself is a composite with cellulose microfibrils embedded in a lignin matrix and that such microfibrils are not oriented perfectly aligned with the fiber axis, and 


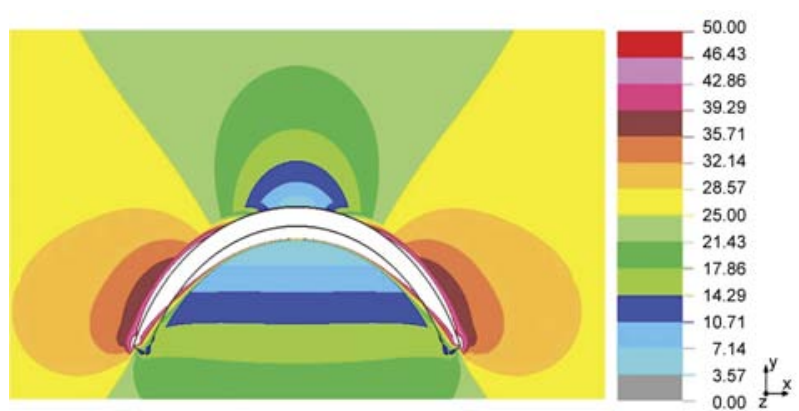

Figure 4. Shows the $\sigma_{\mathrm{xx}}$ stress components distribution around the curved fiber obtained from the finite element analysis

instead, they form a helical pattern along the fiber's length [1].

Figure 4 shows the $\sigma_{\mathrm{xx}}$ distribution and Figure 5 shows the finite element and photoelasticity models, maximum shear stress $\tau_{\max }$, around the curved fiber (seen in white color). As it can be appreciated, the state of stress around the curved fiber is very complex. In Figure 4, it can be seen that the tensile stress $\sigma_{\mathrm{xx}}$ in the matrix and at the fiber matrix interface is positive in the direction of the applied load. But the stress distribution on the convex side is different from the stress distribution on the concave side of the fiber. This tensile stress is acting almost perpendicularly to the fiber ends as shown by the two lobes on the convex sides. A more uniform distribution of $\sigma_{\mathrm{xx}}$ is noted in the matrix, on the concave side of the fiber but still trying to separate the fiber from the matrix, especially at the fiber ends.

Figure 5 shows finite elements and isochromatic fringes from a photoelastic model of a curved fiber embedded in a resin matrix, subjected to a tensile

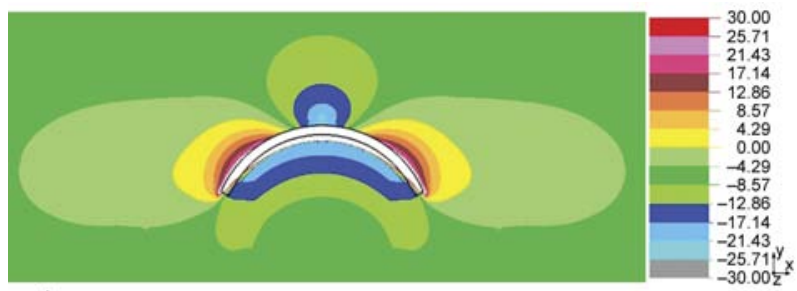

a)

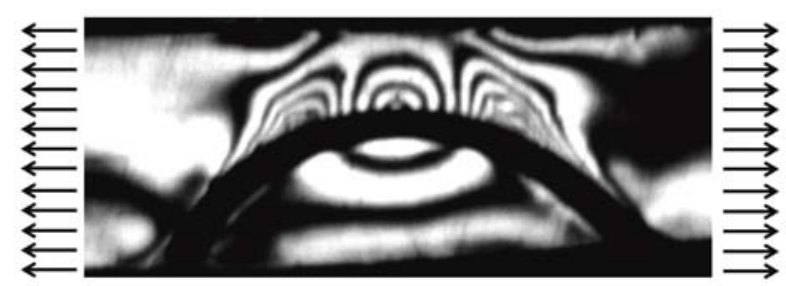

b)

Figure 5. Loci of $\left(\sigma_{1}-\sigma_{2}\right)$, that is, $\tau_{\max }$, the maximum shear stress from (a) finite element and (b) photoelasticity models, around the curved fiber load. Isochromatics are the loci of the points along which the difference in the first and second principal stress remains the same [30]. Thus they are the lines which join the points with equal maximum shear stress magnitude. It should be noticed that the number of fringes on the convex side of the fiber is higher than the number on the concave side. Then, it can be said that the shear stress on the convex side of the curved fiber is different from the shear stress on the concave. The graph (Figure 5b) shows a plot of the shear stress $\tau$ i normalized by the far-field tensile stress $\sigma_{0}$ versus the position along the curved fiber and normalized by the fiber diameter, plotted in a straight line. Along the convex side, three stress discontinuities are observed whereas on the concave side, only one is observed and that their relative magnitude is different but on the concave side is lower. Then, it can be said that, when a fiber is bent the interfacial shear stress induced by the externally applied load will be different along its length depending on its geometry.

Figures 6 to 8 shows plots for the $\sigma_{\mathrm{xx}}, \sigma_{\mathrm{yy}}$ and $\tau_{\max }$ stress components along the fiber-matrix interface on the concave and convex sides of the curved fiber. Figure 6 the normal stress in the center line of the curved fiber in the direction of the applied load. The state of stress and the distribution of the different components on the curved fiber should be discussed considering two different parameter of the composite material. The first is the quality of the fibermatrix interphase and the second is the inherent anisotropy of the natural fiber.

When the level of adhesion between fiber and matrix is low as in the case of the composite made with FIB, the shear stress on both, concave and convex sides will soon produce failure because there is and added stress component contributing to the separation of the fiber from the matrix, that is, $\sigma_{\mathrm{xx}}$ on the concave side especially on the central portion and $\sigma_{\mathrm{yy}}$, although smaller than $\sigma_{\mathrm{xx}}$, still acting to separate fiber from matrix.

When the frictional adhesion is increased as in the case of FIBNA and FIBNAPRE, the amount of polymer penetrating the fiber crevices increases and the upper bound of the interphase strength will be dictated by the shear strength of the matrix. In these cases, there should also be some matrix tearing depending on the degree of entanglement with the outer layer of the fiber. This type of failure could be attributed to the normal stress components at the 


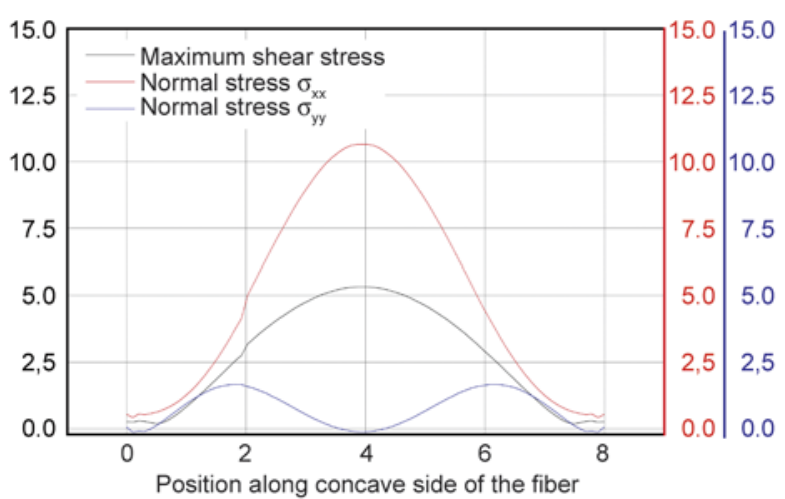

Figure 6. Variation of stress components along the concave side of the curved fiber

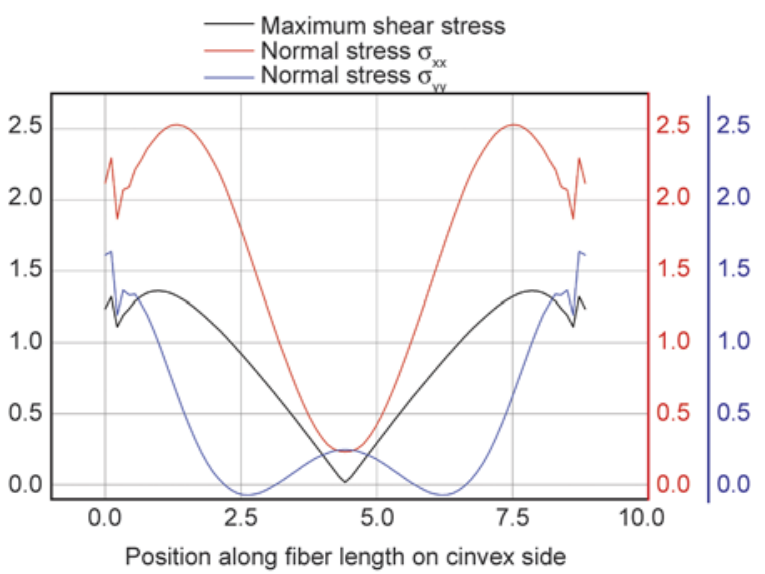

Figure 7. Variation of stress components along the convex side of the curved fiber

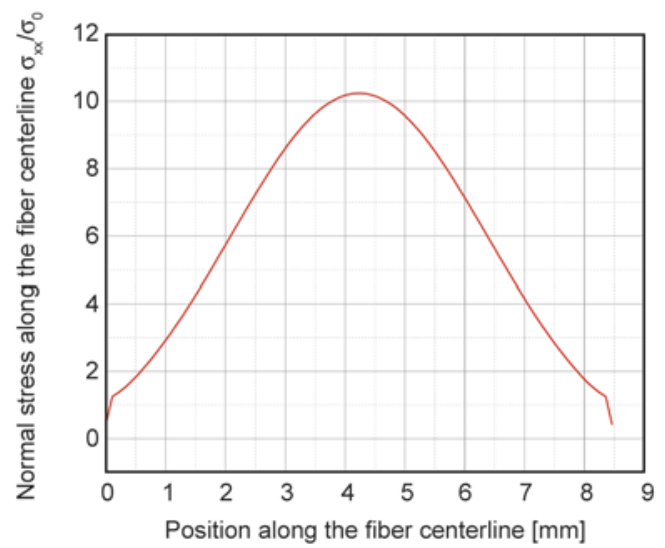

Figure 8. Normal stresses in the center line of the curved fiber in the direction of the applied load

interphase. The formation of covalent bonds in the case of FIBNASIL, contributes to the formation of bridges between fiber and matrix which will better resist the action of both normal and shear stress components.

As seen in Figure 8, the effective length could be considered to be achieved only in the central por- tion of the curved fiber. In fact, it can be seen in Figures 6-8 that the stresses are higher in approximately one third of the total length of the curve fiber, either at the central portion or at both ends. Therefore, the contribution of the fiber to strength will be limited to only portions where the stress transmission is more efficient. It has been reported for jute fibers, that this type of fibers exhibited significant elastic and thermal expansion anisotropy. The fiber's longitudinal Young's modulus $E_{1 \mathrm{f}}$ was estimated to range between 5 and 10 times that of its transverse modulus $E_{2 \mathrm{f}}$ over the temperature range -50 to $50^{\circ} \mathrm{C}$ [31]. A similar behavior is expected for the henequen fibers, at least from the anisotropic point of view. Because of the curvature and depending on the orientation of the bent fiber with respect to the applied load, the fiber's longitudinal Young's modulus $E_{1 \mathrm{f}}$ will form an angle with the applied load. This should reduce the contribution of the fiber to both modulus and strength depending on cosine of the angle $\theta$ formed by fiber longitudinal axis and the direction of the load. Furthermore, when the value of $\theta$ increases, the normal radial component of the applied load also increases in the radial direction of the fiber but its modulus and strength are much lower than the longitudinal value.

These differences in the state of stress along the bent fiber can be explained from a simple force resolution considering the angle between the direction of the applied load and the tangential line on any point along the fiber surface (see Figure 7). One component will be responsible of the interface shear stress, whereas the other component of a normal stress component in a radial direction. It should be noted that the relative magnitude of these stress components will vary from one point to another along the curved fiber. The radial normal stress component should be held responsible for any defibrillation damage to the natural fiber. In the case of FIB, since fiber-matrix adhesion is low, interface debonding is expected and then, no defibrillation damage is observed, but as the adhesion level increases, defibrillation will increase, especially when there is a good fiber wetting. Additionally, the effective length of stress transfer of the bent fiber is not symmetrical nor it is acting on both of its sides. Therefore, this behavior results in a lower stress transfer efficiency as that observed with rigid, stiff fibers which remain straight after processing [32, 33]. 


\subsection{Results from the micromechanical models by Fukuda}

According to the finite element and photoelastic analysis, there seems to be three different portions of reinforcing efficiency of the curved fiber. The inherent anisotropy of the fiber, as shown in Figure 9, can be considered as a composite material with two main material directions. Therefore, considering that the fiber is subjected to different loading conditions, it could be discretized into a number of straight segments, that is, it could be considered equivalent to three different straight fibers with their materials directions according to the curvature. Then, with equivalence if the bent fiber is close to the critical length, it is then possible to consider a ratio $\left(l_{\mathrm{c}} / \bar{l}\right)$ equal to 3 .

Then in Figure 2b, it would result in an asymptotic value for $F\left(l_{\mathrm{c}} / \bar{l}\right)$ of approximately 0.20 . The experimental tensile mechanical properties of the shortnatural fiber composites and the theoretical ones, estimated with the Fukuda-Chou and the FukudaKawata models are shown in Tables 4 and 5. The composite tensile strength was calculated with the Fukuda-Chou model considering two approaches: i) that the natural fiber behaves as straight one, and ii) recognizing their inherent flexibility that curve it during processing. For the calculations, the value of was estimated to be $7.5 \mathrm{~mm}$, and considering an efficiency in one third of the length, the new $\bar{l}$ decreases to $2.5 \mathrm{~mm}$. The corrected values of $F\left(l_{\mathrm{c}} / \bar{l}\right)$ of are used to recalculate the value of the tensile
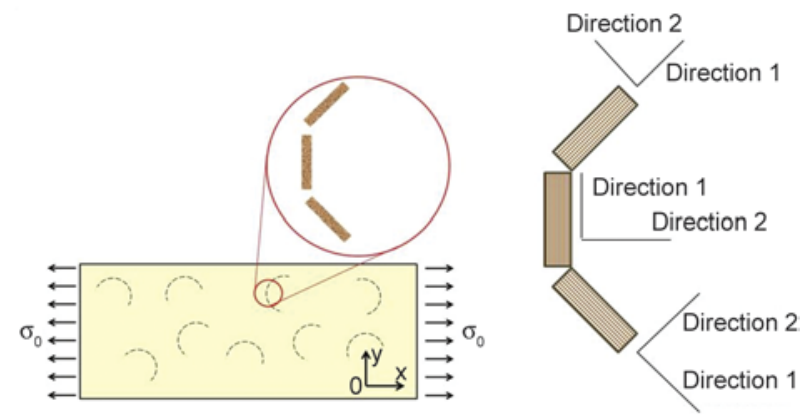

Figure 9. Discretization of a single curved fiber in three straight segments strength. As it can be seen in Table 4 and Figure 10, the theoretical results for the composite strength according to the equation developed by FukudaChou shows better agreement with the experimental results using the corrected effective length due the fiber curvature compared with the direct approach (straight fiber). In another hand, it is evident that there is a better agreement with both approaches for the strong fiber-matrix adhesion composite (FIBNASIL) compared with the other composites, and in fact there is a slight overestimation. It should be remembered that there are factors which are not taken into account in the development of the model. Among them, the stress concentration caused by the early separation of the fibers from the matrix that should results in larger stress concentration factors which are not taken into account anywhere in the theoretical development.

Table 5 shows the values for Young's modulus calculated using Fukuda-Kawata model. In this case the estimated value shows good agreement with the experimental results, but the values were slightly lower than the measured values.

The small inconsistencies found between the experimental and theoretical results could be attributed to the inherent variability in cross-section area, physical and mechanical properties of the natural fibers (a therefore of both, their non-circular cross section and the equivalent diameter), resulting in variations of the fiber aspect ratio and in differences between the experimental and theoretical mechanical properties.

The curvature of natural fibers resulting from their flexibility and the severe stresses during processing

Table 5. Young's modulus calculated utilizing the FukudaKawata model

\begin{tabular}{|l|c|c|c|c|}
\hline & $\begin{array}{c}\text { Experimental } \\
{[\mathbf{M P a}]}\end{array}$ & $\mathbf{C}_{\mathbf{a}}$ & $\mathbf{R}_{\mathbf{0}}$ & $\begin{array}{c}\text { Fukuda-Kawata } \\
{[\mathbf{M P a}]}\end{array}$ \\
\hline FIB & 891 & 0.23 & 0.38 & 837 \\
\hline FIBNA & 945 & 0.23 & 0.38 & 837 \\
\hline FIBNAPRE & 945 & 0.23 & 0.38 & 837 \\
\hline FIBNASIL & 873 & 0.23 & 0.38 & 837 \\
\hline
\end{tabular}

Table 4. Tensile strength calculated utilizing the Fukuda-Chou model

\begin{tabular}{|l|c|c|c|c|c|c|c|}
\hline \multirow{2}{*}{} & \multirow{2}{*}{$\begin{array}{c}\text { Experimental } \\
{[\mathbf{M P a}]}\end{array}$} & \multicolumn{3}{|c|}{ Fukuda-Chou straight fibers } & \multicolumn{3}{|c|}{ Fukuda-Chou curved fibers } \\
\cline { 3 - 8 } & & $\mathbf{C}_{\mathbf{0}}$ & $\mathbf{F}\left(\mathbf{l}_{\mathbf{c}} / \overline{\mathbf{l}}\right)$ & $\begin{array}{c}\text { Tensile strength } \\
{[\mathbf{M P a}]}\end{array}$ & $\mathbf{C}_{\mathbf{0}}$ & $\mathbf{F}\left(\mathbf{I}_{\mathbf{c}} / \overline{\mathbf{l}}\right)$ & $\begin{array}{c}\text { Tensile strength } \\
{[\mathbf{M P a}]}\end{array}$ \\
\hline FIB & 20.80 & 0.20 & 0.38 & 29.60 & 0.05 & 0.2 & 25.30 \\
\hline FIBNA & 21.00 & 0.20 & 0.42 & 30.67 & 0.08 & 0.2 & 25.70 \\
\hline FIBNAPRE & 23.25 & 0.20 & 0.55 & 33.10 & 0.16 & 0.2 & 26.91 \\
\hline FIBNASIL & 27.00 & 0.20 & 0.70 & 35.92 & 0.38 & 0.2 & 30.18 \\
\hline
\end{tabular}




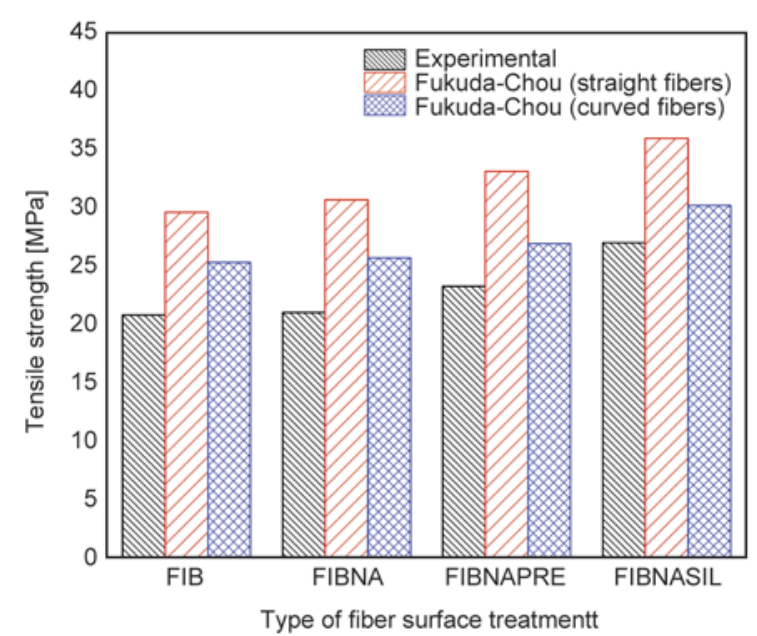

Figure 10. Comparison of the experimental tensile strength with values calculated using the Fukuda-Chou model

is a feature that it is very difficult to control directly but it can be done indirectly by improving the fibermatrix interfacial adhesion. As it has been observed, when the interfacial adhesion is very strong, the critical fiber length is short and the critical fiber aspect ratio also decreases and the fiber fragments tend to remain straight during the processing of the composite. In this case, the fibers with the FIBNASIL surface treatment, the fiber curvature was very low after processing.

\section{Conclusions}

From the photoelastic and finite element analysis, it was seen that the state of stress around a curved fiber inclusion is very complex. It was noticeable that the stress distribution was more uniform in the concave side of the fiber and more complex on the convex side of the curved fiber. It was also noticed that on the convex side of the curved fiber inclusion, there are three distinctive regions along the fiber and that the 'effective fiber length' is very short of approximately one third the average length. The models proposed by Fukuda and based on the probability of length and orientation distribution; they seem to be very convenient to calculate the effective mechanical properties of the composite. This fiber length of higher stress transfer was used to correct the value of the fiber efficiency to recalculate the tensile strength of the composite laminate. Much better agreement was obtained after decreasing the fiber efficiency by using an average length value to compensate for the fiber curvature in the strength properties specially, also, the best agreement between the predicted and the experimental values was observed with the fiber-matrix system subjected to surface treatment with both mechanical and chemical bonding being this the best combination to get the most effective mechanical properties of the composite. Then, prediction of the tensile mechanical properties using the micromechanical models proposed by Fukuda-Kawata and coworkers, would be reasonable if the curved fiber is considered equivalent to two or three shorter straight fiber fragments to estimate the reinforcing efficiency in the composite.

\section{Acknowledgements}

The authors would like to thank Consejo Nacional de Ciencia y Tecnología for the grant through project U42432-K and Mr. Miguel A. Escalante-Solís is grateful for the doctoral scholarship granted to him.

\section{References}

[1] Herrera-Franco P. J., Valadez-Gonzalez A.: Mechanical properties of continuous natural fibre-reinforced polymer composites. Composites Part A: Applied Science and Manufacturing, 35, 339-345 (2004).

DOI: 10.1016/j.compositesa.2003.09.012

[2] Ku H., Wang H., Pattarachaiyakoop N., Trada M.: A review on the tensile properties of natural fiber reinforced polymer composites. Composites Part B: Engineering, 42, 856-873 (2011).

DOI: $10.1016 /$ j.compositesb.2011.01.010

[3] Sawpan M. A., Pickering K. L., Fernyhough A.: Effect of fibre treatments on interfacial shear strength of hemp fibre reinforced polylactide and unsaturated polyester composites. Composites Part A: Applied Science and Manufacturing, 42, 1189-1196 (2011).

DOI: $10.1016 /$ j.compositesa.2011.05.003

[4] Yue C. Y., Cheung W. L.: Interfacial properties of fibrereinforced composites. Journal of Materials Science, 27, 3843-3855 (1992).

DOI: $10.1007 / \mathrm{BF} 00545467$

[5] Anumandla V., Gibson R. F.: A comprehensive closed form micromechanics model for estimating the elastic modulus of nanotube-reinforced composites. Composites Part A: Applied Science and Manufacturing, 37, 2178-2185 (2006).

DOI: 10.1016/j.compositesa.2005.09.016

[6] Kalaprasad G., Joseph K., Thomas S., Pavithran C.: Theoretical modelling of tensile properties of short sisal fibre-reinforced low-density polyethylene composites. Journal of Materials Science, 32, 4261-4267 (1997).

DOI: $10.1023 / \mathrm{A}: 1018651218515$ 
[7] George J., Sreekala M. S., Thomas S.: A review on interface modification and characterization of natural fiber reinforced plastic composites. Polymer Engineering and Science, 41, 1471-1485 (2001).

DOI: $10.1002 /$ pen. 10846

[8] Bowyer W. H., Bader M. G.: On the re-inforcement of thermoplastics by imperfectly aligned discontinuous fibres. Journal of Materials Science, 7, 1315-1321 (1972).

DOI: $10.1007 / \mathrm{BF} 00550698$

[9] Nairn J. A.: Exact and variational theorems for fracture mechanics of composites with residual stresses, traction-loaded cracks, and imperfect interfaces. International Journal of Fracture, 105, 243-271 (2000).

DOI: 10.1023/A:1007666426275

[10] Simonsen J.: Efficiency of reinforcing materials in filled polymer composites. Forest Products Journal, 47, 74-81 (1997).

[11] Doan T-T-L., Gao S-L., Mäder E.: Jute/polypropylene composites I. Effect of matrix modification. Composites Science and Technology, 66, 952-963 (2006).

DOI: 10.1016/j.compscitech.2005.08.009

[12] Beckermann G. W., Pickering K. L.: Engineering and evaluation of hemp fibre reinforced polypropylene composites: Fibre treatment and matrix modification. Composites Part A: Applied Science and Manufacturing, 39, 979-988 (2008).

DOI: $10.1016 /$ j.compositesa.2008.03.010

[13] Herrera-Franco P. J., Valadez-Gonzalez A.: A study of the mechanical properties of short natural-fiber reinforced composites. Composites Part B: Engineering, 36, 597-608 (2005).

DOI: 10.1016/j.compositesb.2005.04.001

[14] Fukuda H., Kawata K.: On Young's modulus of short fibre composites. Fibre Science and Technology, 7, 207222 (1974).

DOI: $10.1016 / 0015-0568(74) 90018-9$

[15] Fukuda H., Chou T-W.: A probabilistic theory for the strength of short fibre composites. Journal of Materials Science, 16, 1088-1096 (1981).

DOI: $10.1007 / \mathrm{BF} 00542756$

[16] Joffe R., Andersons J., Wallström L.: Strength and adhesion characteristics of elementary flax fibres with different surface treatments. Composites Part A: Applied Science and Manufacturing, 34, 603-612 (2003). DOI: 10.1016/S1359-835X(03)00099-X

[17] Abdelmouleh M., Boufi S., ben Salah A., Belgacem M. N., Gandini A.: Interaction of silane coupling agents with cellulose. Langmuir, 18, 3203-3208 (2002).

DOI: $10.1021 / \mathrm{la} 011657 \mathrm{~g}$

[18] Brochier M-C., Abdelmouleh S., M., Boufi S., Belgacem M. N., Gandini A.: Silane adsorption onto cellulose fibers: Hydrolysis and condensation reactions. Journal of Colloid and Interface Science, 289, 249261 (2005).

DOI: $10.1016 /$ j.jcis.2005.03.070
[19] Valadez-Gonzalez A., Cervantes-Uc J. M., Olayo R., Herrera-Franco P. J.: Effect of fiber surface treatment on the fiber-matrix bond strength of natural fiber reinforced composites. Composites Part B: Engineering, 30, 309-320 (1999). DOI: 10.1016/S1359-8368(98)00054-7

[20] Procopio A. T., Zavaliangos A., Cunningham J. C.: Analysis of the diametrical compression test and the applicability to plastically deforming materials. Journal of Materials Science, 38, 3629-3639 (2003).

DOI: $10.1023 / \mathrm{A}: 1025681432260$

[21] Herrera-Franco P. J., Drzal L. T.: Comparison of methods for the measurement of fibre/matrix adhesion in composites. Composites, 3, 2-27 (1991).

DOI: 10.1016/0010-4361(92)90282-Y

[22] Monette L., Anderson M. P., Grest G. S.: The meaning of the critical length concept in composites: Study of matrix viscosity and strain rate on the average fiber fragmentation length in short-fiber polymer composites. Polymer Composites, 14, 101-105 (1993).

DOI: $10.1002 / p c .750140204$

[23] Joseph K., Thomas S., Pavithran C., Brahmakumar M.: Tensile properties of short sisal fiber-reinforced polyethylene composites. Journal of Applied Polymer Science, 47, 1731-1739 (1993).

DOI: 10.1002/app.1993.070471003

[24] Newman R. H., Hebert P., Dickson A. R., Even D., Fernyhough A., Sandquist D.: Micromechanical modelling for wood-fibre reinforced plastics in which the fibres are neither stiff nor rod-like. Composites Part A: Applied Science and Manufacturing, 65, 57-63 (2014). DOI: 10.1016/j.compositesa.2014.05.012

[25] van Hattum F. W., Bernardo C. A.: A model to predict the strength of short fiber composites. Polymer Composites, 20, 524-533 (1999).

DOI: $10.1002 / p c .10376$

[26] Venkateshwaran N., ElayaPerumal A.: Modeling and evaluation of tensile properties of randomly oriented banana/epoxy composite. Journal of Reinforced Plastics and Composites, 30, 1957-1967 (2011).

DOI: $10.1177 / 0731684411430559$

[27] Ulrych F., Sova M., Vokrouhlecky J., Turcic B.: Empirical relations of the mechanical properties of polyamide 6 reinforced with short glass fibers. Polymer Composites, 14, 229-237 (1993).

DOI: $10.1002 /$ pc. 750140308

[28] Jones R. M.: Mechanics of composite materials. Taylor and Francis, Miami (1998).

[29] Tucker C. L., Liang E.: Stiffness predictions for unidirectional short-fiber composites: Review and evaluation. Composites Science and Technology, 59, 655671 (1999).

DOI: $10.1016 / \mathrm{S} 0266-3538(98) 00120-1$

[30] Dally J. W., Riley W. F.: Experimental stress analysis. McGraw-Hill, New York (1991). 
[31] Cichocki Jr. F. R., Thomason J. L.: Thermoelastic anisotropy of a natural fiber. Composites Science and Technology, 62, 669-678 (2002).

DOI: $10.1016 / \mathrm{S} 0266-3538(02) 00011-8$

[32] Cazaurang-Martinez M. N., Herrera-Franco P. J., Gonzalez-Chi P. I., Aguilar-Vega M.: Physical and mechanical properties of henequen fibers. Journal of Applied Polymer Science, 43, 749-756 (1991).

DOI: 10.1002/app.1991.070430412
[33] Manikandan K. C., Diwan S. M., Thomas S.: Tensile properties of short sisal fiber reinforced polystyrene composites. Journal of Applied Polymer Science, 60, 1483-1497 (1996).

DOI: $10.1002 /($ SICI)1097-4628(19960531)60:9<1483 :AID-APP23>3.0.CO;2-1 